\title{
Resting state fMRI entropy probes complexity of brain activity in adults with ADHD
}

Moses Sokunbi ${ }^{\text {ab*}}$, Wilson Fung ${ }^{\mathrm{c}}$, Vijay Sawlani ${ }^{\mathrm{d}}$, Sabine Choppin ${ }^{\mathrm{abf}}$, David Linden ${ }^{\mathrm{ab}}$, Johannes Thome ${ }^{\mathrm{e}}$

${ }^{a}$ MRC Centre for Neuropsychiatric Genetics and Genomics, Institute of Psychological Medicine and Clinical Neurosciences, Cardiff School of Medicine, Cardiff University, Cardiff, UK.

Imaging Science, Cardiff University Brain Research Imaging Centre (CUBRIC), Cardiff University, Cardiff, UK.

${ }^{c}$ Neuroscience and Molecular Psychiatry, Institute of Life Science, College of Medicine, Swansea University, Swansea, UK.

${ }^{\mathrm{d}}$ Department of Radiology, The New Queen Elizabeth Hospital, Birmingham University Hospitals, Birmingham, UK.

e Department of Psychiatry, University of Rostock, Gehlsheimer Straße 20, D-18147 Rostock, Germany.

${ }^{\mathrm{f}}$ Department of Psychiatry, Centre Hospitalier Guillaume Régnier, Université Rennes 1, Rennes, France.

*Corresponding author.

Cardiff University Brain Research Imaging Centre (CUBRIC),

Cardiff University,

70 Park Place,

Cardiff CF10 3AT

United Kingdom. 
Telephone: +442920870077

E-mail address: SokunbiMO@,cardiff.ac.uk

\begin{abstract}
In patients with attention deficit hyperactivity disorder (ADHD), quantitative neuroimaging techniques have revealed abnormalities in various brain regions, including the frontal cortex, striatum, cerebellum, and occipital cortex. Nonlinear signal processing techniques such as sample entropy have been used to probe the regularity of brain magnetoencephalography signals in patients with ADHD. In the present study, we extend this technique to analyse the complex output patterns of the 4 dimensional resting state functional magnetic resonance imaging signals in adult patients with ADHD. After adjusting for the effect of age, we found whole brain entropy differences $(P=0.002)$ between groups and negative correlation $(r=-$ 0.45) between symptom scores and mean whole brain entropy values, indicating lower complexity in patients. In the regional analysis, patients showed reduced entropy in frontal and occipital regions bilaterally and a significant negative correlation between the symptom scores and the entropy maps at a family-wise error corrected cluster level of $P<0.05$ $(P=0.001$, initial threshold). Our findings support the hypothesis of abnormal frontalstriatal-cerebellar circuits in ADHD and the suggestion that sample entropy is a useful tool in revealing abnormalities in the brain dynamics of patients with psychiatric disorders.
\end{abstract}

Keywords: ADHD; BOLD; complexity; fMRI; Sample entropy

\title{
1. Introduction
}

Attention Deficit Hyperactivity Disorder (ADHD) is one of the most common mental disorders of childhood, affecting $5-10 \%$ of all children. It frequently persists into 
adolescence, or even adulthood (Biederman, 1998), with a prevalence of approximately $4 \%$ (Biederman, 2005). Its core clinical symptoms include inattention, hyperactivity and impulsivity with the attentional deficit being usually the most functionally impairing symptom in adults (Barkley, 2003). Although the pathophysiology of ADHD remains unclear, some studies reported that the neural basis of this disorder resides mainly in anatomical and functional disturbances of frontal-striatal-cerebellar circuits (Giedd et al., 2001).

Many functional neuroimaging techniques have been explored to study the pathophysiology underlying ADHD. These techniques include single photon emission computed tomography (SPECT), positron emission tomography (PET) and blood oxygenation level dependent (BOLD) functional MRI (fMRI). Most SPECT and PET studies have probed the brain's resting-state to study the pathophysiology of ADHD. These studies reported abnormalities in the frontal cortex (Lee et al., 2005), striatum (Lou et al., 1990), anterior cingulate cortex (ACC) (Langleben, 2002), sensorimotor cortex (SMC) (Lee et al., 2005), occipital cortex ( Schweitzer et al., 2003) and cerebellum (Lee et al., 2005). Task specific BOLD fMRI studies of ADHD have also been implemented but the results were found to be inconsistent. Both hypofrontality (Rubia et al., 1999) and hyperfrontality (Schulz et al., 2004) have been reported. The discrepancy could be a result of different tasks, ages and comorbidity in the studies. However, even studies using very similar tasks (Schulz et al., 2004; Tamm et al., 2004) have produced divergent results. Subtle differences in tasks and patients' performance strategies between the two studies might partly account for the discrepancies. Hence, it may be difficult to extract reliable markers of ADHD pathophysiology from task-based studies. 
The resting-state (Raichle et al., 2001) fMRI approach has therefore been introduced as an alternative perspective on brain functional abnormalities in ADHD. Resting-state BOLD fMRI data allow for the analysis of functional connectivity patterns in brain networks and the temporal dynamics of their activity fluctuations (Rotarska-Jagiela et al., 2010). In children with ADHD, several studies have reported decreased amplitude of low-frequency fluctuations (ALFF) in the right inferior frontal cortex, the bilateral cerebellum and the vermis as well as increased ALFF in the right anterior cingulate cortex (Zang et al., 2007). Abnormal dorsal anterior cingulate cortex (dACC) functional connectivity patterns have been reported in adolescent ADHD patients (Tian et al., 2006). ADHD patients exhibited decreased regional homegeneity (ReHo) in frontal-striatal-cerebellar circuits but increased ReHo in the occipital cortex (Cao, 2006). Very few studies have applied this method to adult ADHD, but results of a study employing kernel principal component analysis to discriminate adults with ADHD from normal controls (Wang et al., 2011) are encouraging.

Nonlinear signal processing techniques such as approximate entropy (ApEn) (Pincus, 1991; Pincus, 1995) and sample entropy (SampEn) (Richman and Moorman, 2000) provide a relatively new measure to probe the complexity of brain fMRI dynamics (Sokunbi et al., 2011). Entropy values reflect the number of times the patterns in a signal are repeated and thus measure the randomness and predictability of a stochastic process and in general increase with greater randomness. A lower value of SampEn thus indicates lower complexity of the signal or system. Recently, Gomez et al. (Gomez et al. 2011) applied SampEn to analyse the spontaneous MEG activity in ADHD patients. They performed five minutes of recording with a 148-channel whole-head magnetometer in 14 ADHD patients and 14 control subjects. They found that the SampEn values of the ADHD patients' MEGs were lower than those of the controls. Also, there were statistically significant differences $(\mathrm{p}<0.01$, Student's 
t-test with Bonferoni's correction) at the five analysed brain areas: anterior, central, posterior, left lateral and right lateral. Until now the complex output patterns of the 4D blood oxygen level dependent (BOLD) signals in ADHD have remained unexplored.

The rationale for studying resting state data of psychiatric patients with complexity measures is that complex output patterns of a system can give an indication of the health and robustness of the system (Goldberger et al., 2002a). Therefore, the characterization and analysis of the brain's output in terms of its complexity may reveal a better understanding of an individual's adaptive capacity, the ability to respond to unpredictable perturbations and stresses, which is presumed to be impaired in mental disorders. Complex organic systems such as the human brain have evolved to maximise adaptive capacity (Wolf and Linden, 2012). The degradation of these processes with age and disease is associated with loss of complexity in the dynamics of complex physiological systems (Lipsitz, 2004). Chaotic and complex behaviours indicate a healthy system whereas more predictable behaviours would be linked to pathological states (Pool, 1989).

The aim of the present study was to investigate differences in the complex output patterns of resting state fMRI signals in adult ADHD patients when compared to age-matched healthy controls. Current models of ADHD posit funcitonal deficits in the frontal cortex, striatum, cerebellum, and occipital cortex of the brain (Seidman et al., 1998; Swanson et al., 1998). We expected the functional physiological complexity of ADHD patients (as reflected in the entropy values) to be reduced, and this reduction to scale with symptom severity. 


\section{Materials and Methods}

\subsection{Participants}

We recruited 17 ADHD patients (8 female, mean (standard deviation; SD) age 29.65 ( \pm 10.19$)$ ) from National Health Service (NHS) out-patient clinics in Swansea and 13 age- and gender-matched controls (8 female, mean (SD) age $29.69( \pm 8.39)$ ). The study was approved by the South West Wales Research Ethics Committee. Exclusion criteria were inability to give informed consent and MRI contraindication.

A detailed written and verbal explanation of the purpose and design of the study was provided to all the participants and written informed consent obtained prior to the commencement of the study.

Patients were receiving psychotropic medication for ADHD at the time of scanning (dose): Methylphenidate 32 mg (6 patients), D-amphetamine 70mg (2 patients), Atomoxetine 80mg (1 patient), (Risperidone $0.5 \mathrm{mg}$, (1 patient), and benzodiazepines (1 patient). One patient was treated with Acamprosate $666 \mathrm{mg}$ for alcohol dependence and was currently abstinent. Three patients were additionally treated with antidepressant medication. Five patients were not on any medication.

All patients and control participants completed the Conners' Adult ADHD Rating Scales (CAARS), a standardized self-rating for adults undergoing evaluation for ADHD (Conners et al., 1999a; Conners et al., 1999b; Erhardt et al., 1999). CAARS are a set of easily administered instruments consisting of self-reports and observer ratings allowing for the multimodal assessment of adult ADHD symptoms and behaviours. The CAARS subscales include inattention/memory problems, hyperactivity/restlessness, impulsivity/emotional 
liability, problems with self-concept and ADHD index. The mean ( $\pm 2 \mathrm{SD})$ ADHD score for the control and ADHD groups are listed in Table 1.

\subsection{Resting state fMRI acquisition}

Functional MR images were acquired with a $\mathrm{T}_{2} *$ weighted gradient echo echo-planar imaging sequence (EPI) in the axial plane using a GE Medical Systems twin-speed Signa HDx 3T MRI scanner. A total of 31 axially orientated $4 \mathrm{~mm}$ thick contiguous sequential slices were obtained for each of 100 volumes using a TR of $3000 \mathrm{~ms}$, TE of $35 \mathrm{~ms}$, flip angle of $90^{\circ}$, field of view of $240 \times 240 \mathrm{~mm}$ and matrix $64 \times 64$. The first three volumes were discarded to allow for transient effects. The fMRI images were acquired using a 16 channels head coil without a task or stimulus ('resting state').

\subsection{Image pre-processing}

The image pre-processing was performed on the fMRI data using version 8 of Statistical Parametric Mapping software (SPM8; The Wellcome Department of Imaging Neuroscience, UCL, London, UK). The fMRI data were realigned using Realign (Est \& Res) from the Spatial pre-processing section of SPM8 to correct for head movement distortion. Temporal high pass filtering (128 seconds) was performed from Specify $1^{\text {st }}$-level of the model specification, review and estimation section to reduced low frequency noise. Each voxel time series was standardized to a mean of zero and standard deviation of unity to allow data set with different amplitudes to be compared (Richman and Moorman, 2000).

\subsection{Estimation of input parameters for calculating SampEn}

Richman and Moorman (2000) developed sample entropy (SampEn) from the modification of the approximate entropy (ApEn) algorithm (Pincus, 1991; Pincus, 1995) to reduce the bias of ApEn, where self-matches were excluded from the ApEn algorithm, $(i \neq j)$ and $(1 \leq i \leq N-m)$ 
i.e. vectors are not compared to themselves. SampEn has the advantage of being less dependent on time series length, and showing relative consistency over a broader range of possible $\mathrm{r}$, $\mathrm{m}$, and $\mathrm{N}$ values under circumstances where ApEn does not (Richman and Moorman, 2000). SampEn is the negative logarithm of the conditional probability that two sequences remain similar at the next point, where self matches are not included in calculating the probability.

The same input parameters of $\mathrm{m}, \mathrm{r}, \mathrm{N}$ and $\boldsymbol{\tau}$ used in calculating ApEn are also applicable to SampEn. The SampEn for a given $\mathrm{N}$-dimensional time series $\left(x_{1}, x_{2}, \ldots \ldots, x_{N}\right)$ is defined as:

$$
\begin{aligned}
& \operatorname{SampEn}(m, r, N)=-\ln \left[\frac{U^{m+1}(r)}{U^{m}(r)}\right] \\
& U^{m}(r)=[N-m \tau]^{-1} \sum_{i=1}^{N-m \tau} C_{i}^{m}(r)
\end{aligned}
$$

Where

$$
\begin{gathered}
C_{i}^{m}(r)=\frac{B_{i}}{N-(m+1) \tau} \\
B_{i}=\text { number of } \mathrm{j} \text { where } \mathrm{d}\left|X_{i}, X_{j}\right| \leq r \\
X_{i}=\left(x_{i}, x_{i+\tau}, \ldots ., x_{i+(m-1) \tau}\right) \\
X_{j}=\left(x_{j}, x_{j+\tau}, \ldots . ., x_{j+(m-1) \tau}\right) \\
1 \leq j \leq N-m \tau, j \neq i
\end{gathered}
$$

In equation (1), $N$ is the number of time points, $m$ specifies the pattern length, 
$\mathrm{r}$ defines the tolerance value and $\tau$ is the time delay. The two patterns $i$ and $j$ of $\mathrm{m}$ measurements of the time series are similar if the difference, $\mathrm{d}\left|X_{i}, X_{j}\right|$ between any pair of corresponding measurements of $X_{i}$ and $X_{j}$ is less than or equal to r, as shown in equation (2). $X_{i}$ and $X_{j}$ are m-dimensional pattern vectors whose components are time delayed versions of the elements in the original time series with time delay, $\tau$ as shown in equation (3) and (4).

Most SampEn analysis of biomedical signals have used $m=2$ and $0.1 \leq r \leq 0.50$ (Costa et al. 2002; Norris et al. 2009; Yang et al. 2011; Catarino et al. 2011; Mizuno et al. 2010; Takahashi et al. 2009, 2010; Protzner et al. 2010), a few have used $\mathrm{m}=1$ and $0.1 \leq \mathrm{r} \leq 0.35$ (Escudero et al. 2006; Yang et al 2013). Since there are no rigorous guidelines for choosing parameters to calculate SampEn for biomedical signals and most of the parameters that have been chosen appear to be data specific, we investigated the parameters that would be appropriate for calculating SampEn for our fMRI analysis. In order to estimate appropriate and robust values of $m$ and $r$ for the computation of SampEn, we evaluated the ability of SampEn to discriminate patients with ADHD from healthy controls using the value of the area of receiver operating characteristic (ROC) curve (Zweig and Campbell, 1993). The area under the ROC curve is used as a guide to classify the precision of a diagnostic test. ROC area with values between 0.90 and 1 means the precision of the diagnostic test is excellent, values between 0.80 and 0.89 means the test is good, fair if the results are between 0.70 and 0.79 , poor when the value of the ROC area is between 0.60 and 0.69 and bad for values ranging from 0.50 to 0.59 . We computed the mean whole brain SampEn values for each of the 17 adult patients with ADHD and 13 control subjects with $\mathrm{N}=97$ of fMRI time series, $\mathrm{m}=$ 1 and $\mathrm{m}=2, \tau=1$ and $\mathrm{r}$ ranging from 0.02 to 0.7 at intervals of 0.04 . For $\mathrm{m}=1$, only $\mathrm{r}$ values 
of 0.10 to 0.7 returned mean whole brain SampEn values, while for $m=2$, only $r$ values of 0.26 to 0.7 retuned SampEn values. The value of the ROC area of the mean whole brain SampEn for each $r$ value for $m=1$ and $m=2$ was calculated using SPSS software. A plot of the value of the ROC area for each $r$ value for $m=1$ and $m=2$ is shown in Fig. 1 . The plot shows that the values of the ROC area for SampEn are good for all $r$ values for both $m=1$ and $m=2$. For $\mathrm{m}=1$, the minimum ROC area of 0.819 occurs at $r=0.38$ and the maximum ROC area of 0.850 occurs at $r=0.10$ and $r=0.26$. For $m=2$, the minimum ROC area of 0.846 occurred $0.58 \leq \mathrm{r} \leq 0.70$ and the maximum ROC area of 0.851 occurred at $r=0.46$. Based on our findings, we therefore chose $\mathrm{m}=2$ and $\mathrm{r}=0.46$ as the appropriate parameters for our study.

\subsection{Calculation of SampEn for 3-dimensional (3D) fMRI data}

Using the approach developed by Sokunbi et al. (2011), whole brain SampEn maps for each individual of both groups were generated on a voxel by voxel basis on a MATLAB and C platform using the SampEn algorithm. The parameters used for calculating SampEn were $\mathrm{N}=$ 97 of fMRI time series, $\mathrm{m}=2, \tau=1$ (to reduce autocorrelation) and $\mathrm{r}=0.46$. The calculation of SampEn on the whole brain was threshold at 0.1 times the maximum signal to exclude voxels being calculated outside the brain. The mean SampEn value for the whole brain was calculated. Whole brain SampEn maps were generated and normalised to a standard echo planar imaging (EPI) template. Fig. 2 depicts the 3D SampEn maps of the whole brain for

participants of the ADHD and control groups respectively, having the same age. Spatial smoothing was also performed using the full-width at half maximum (FWHM) of the

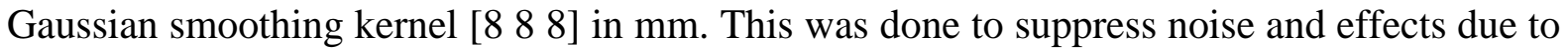
residual differences in functional and gyral anatomy during inter-subject averaging. 


\subsection{Statistical analysis}

Associations between the symptom (ADHD) score and SampEn were tested on a global and regional basis. Parametric statistical analysis was performed on the mean whole brain SampEn measures of each subject in both groups using the Statistical Package for Social Sciences (SPSS 16.0; Chicago, IL, USA) software. The regional analysis was performed using the two-sample t-test in SPM 8, comparing the SampEn maps of the ADHD and control groups. Correlations between the SampEn maps and the ADHD score were then found by using the standard SPM approach from the second-level group analysis.

\section{Results}

The participants' characteristics and SampEn measurements are shown in Table 1. The mean whole brain SampEn values for the ADHD group was significantly lower than for the control group (1.04 \pm 0.10 vs. $1.14 \pm 0.07, \mathrm{t}=3.427, P<0.002)$. After adjusting for the effect of age, using the General Linear Model in SPSS, the mean whole brain SampEn of both groups remained significantly different $(P=0.002)$. This is illustrated in Fig. 3(A). The mean ADHD score of both groups is also significantly different $(P=0.000)$. There was a significant ( $P=0.012)$ negative correlation $(\mathrm{r}=-0.45)$ between the mean ADHD score and the mean whole brain SampEn for the whole group (ADHD and control). Fig. 3(B) shows how the mean whole brain SampEn reduces with increase in the ADHD score.

When the regional analysis was performed with family-wise error (FWE) corrected cluster level significance (Nichols and Hayasaka, 2003) of $P<0.05$, the result of the two-sample t-test $(P=0.001)$ after correcting for age is shown in Fig. 4. The discriminated regions in yellow in the rendered image show significantly lower SampEn in, the ADHD group. The error bar plot of these identified regions show complete separation between the groups (Fig. 4). Table 2 
shows the anatomical location of the regions shown in Fig. 4 of both white and grey matter tissue types. The association between the ADHD score and SampEn was investigated regionally at a corrected cluster level significance of $P<0.05$ while performing a multiple regression analysis $(P=0.001)$ of the SPM second-level group analysis. The result shows that SampEn correlates negatively with the ADHD score i.e. SampEn reduces with increases in the ADHD score. Fig. 5 shows the axial image slices of regions in the brain showing significant negative correlation between the ADHD score and SampEn of both groups. Fig. 6 (A) - (F) depicts the association between SampEn and ADHD score at; (A) Middle Occipital Gyrus; (B) Cuneus; (C) Middle Temporal Gyrus; (D) Postcentral Gyrus; (E) Precuneus; and (F) Medial Frontal Gyrus. As for the global value, the regional SampEn values negatively correlated with the ADHD scores. Table 3 shows the list of the peak regions found from the significant negative correlation between the ADHD score and SampEn.

\section{Discussion}

We show a reduction in the complexity of resting brain activity, as measured by signal entropy (SampEn), in adult ADHD patients compared to healthy and age-matched controls. In order to reflect the continuous distribution of ADHD symptoms in the population we conducted a correlation analysis between symptom scores and entropy values, which revealed increasing symptom load with decreasing complexity of brain activity. This lower signal entropy is consistent with the Goldberger/Lipsitz model for robustness (Goldberger, 1996; Goldberger, 1997; Goldberger et al., 2002a; Goldberger et al., 2002b) where ill health and less robust systems exhibit less complexity (lower entropy) in their physiological output. In the present study, we have shown for the first time that this can be extended to BOLD fMRI data acquired from ADHD patients. 
Our results are consistent with those of Gomez et al. (Gomez et al., 2011) who found reduced SampEn in the spontaneous MEG activity of 14 adult ADHD patients compared to controls. Our findings also agree with previous research works that have applied other measures of entropy such as approximate entropy (ApEn) to estimate the complexity of ADHD patients from EEG recordings (Sohn et al., 2006). However, we could avail ourselves of the superior spatial resolution and fidelity of fMRI compared to non-invasive electrophysiology and identified hotspots of reduced complexity in several neocortical (mainly frontal and occipital) and limbic (anterior cingulate) areas.

The discriminated brain regions listed in Table 2 and 3 are a part of the frontal-striatalcerebellar circuits implicated in network models of ADHD pathology. In a resting state fMRI study, Cao and colleagues, using a regional/network homogeneity approach, also found a deficit in the anterior cingulate region of ADHD patients when compared to normal controls (Cao et al., 2006). Moreover, Uddin et al. (Uddin et al., 2008) found a deficit in the precuneus of adult ADHD patients when compared to healthy controls using the same approach. Decreased functional connectivity between the anterior cingulate gyrus and precuneus in ADHD patients was observed using a seed based correlational approach (Castellanos et al., 2008). The precuneus and anterior cingulate are parts of the so-called default-mode network (Raichle et al., 2001), which has also been implicated in ADHD (Uddin et al., 2008; Cao et al., 2006), and both these areas are also highlighted by the present study. The areas where we identified reduced signal complexity also conform to several other previous studies. For example, using the small world network approach, Wang et al. (Wang et al., 2009) reported decreased efficiency of network nodes in the middle temporal gyrus of ADHD patients with respect to normal controls. In magnetic resonance (MR) studies of brain structure, using a manual and semi-automated region of interest (ROI) approach, Hill et al. (Hill et al., 2003) 
found a reduction in the superior frontal gyrus of ADHD patients when compared to normal controls. Also, a similar structural study found a reduction in the total brain structure and occipital lobe of ADHD patients (Castellanos et al., 2001; Castellanos et al., 2002).

The extant functional and structural imaging studies thus suggest abnormalities in specific brain regions of ADHD patients. However, the mechanism by which a structural change influences functional activity and whether these changes occur at a specific location or across a network is unclear. In the present study we have successfully shown that complexity using measures of signal entropy (SampEn) is a proxy for the ADHD pathology.

The selection of the input parameters for calculating SampEn based on the value of the ROC area (Fig. 1) showed that SampEn demonstrated good ability in discriminating adult ADHD patients from age-matched healthy controls both for $m=1$ and $m=2$ in the tolerance range $0.1 \leq r \leq 0.70$. This may suggest why previous studies using SampEn either with $m=1$ or $m=2$ obtained good results (Escudero et al. 2006; Yang et al 2013; Costa et al. 2002; Norris et al. 2009; Yang et al. 2011; Catarino et al. 2011; Mizuno et al. 2010; Takahashi et al. 2009, 2010; Protzner et al. 2010). Fig. 1 showed that the ROC area obtained over the tolerance range $0.1 \leq \mathrm{r} \leq 0.70$ using $\mathrm{m}=2$ is more consistent than $\mathrm{m}=1$, though $\mathrm{m}=1$ produced more tolerance values for comparison. The discrimination obtained from the ROC analysis is by no means confined to these parameters and can be obtained via a wide range of parameters; these are just the optimal ones (Fig. 1). Future studies with independent data sets are needed to determine whether these parameters are optimal generally, or just for this dataset. To avoid a significant contribution from noise in the computation of entropy, one must choose $\mathrm{r}$ larger than most of the noise (Pincus, 1991). The high tolerance value, $r=0.46$ we have used in this 
study is a good choice because it shows better robustness to reduced noise in distinguishing between the system complexities of adult patients with ADHD and the control group.

The limitations of the present study include a short number of time points (97 volumes) of fMRI data, the small patient sample size and the possible medication effects. A higher number of time points correspond to higher SampEn values which enable a better approximation of the measured entropy. Previous work has demonstrated functional connectivity differences also in unmedicated ADHD patients (Sun et al., 2012), and the changes observed after dopaminergic medication are different from the group differences observed here (Wilson et al., 2013). There is no clear indication of medication effect on the measurement of SampEn. The relationship between medication effect and SampEn may be further explored in future studies. What is attractive about the current study is that we demonstrate clear and clinically relevant group differences on a measure that can be obtained in a clinical scanning suite in under ten minutes.

\section{Conclusion}

Our analysis portrays a novel implementation of temporal signal entropy (SampEn) to investigate the changes in complexity of 4D brain fMRI signals in ADHD patients when compared to healthy controls. We found reduced complexity especially in the activity of frontal and occipital regions and in parts of the "default-mode network”, and reduced global and local complexity correlated with symptom severity. We suggest that SampEn is a useful and easily obtainable measure to reveal changes in ADHD brain dynamics. 


\section{Acknowledgments}

We would like to thank Sian Roberts (MRI Superintendent), Singleton Hospital, Swansea and Gareth Evans (MRI Superintendent) Singleton and Morriston Hospitals, Swansea for their efforts in the acquisition of the resting state fMRI data and Dr Matthew Pugh for help with patient recruitment and assessments. Johannes Thome has obtained financial support (e.g. lecture honoraria, grants for research projects, and scientific meetings, advisory-board membership) from AstraZeneca, Bristol-Meyers Squibb, Ever Neuro Phama, Janssen-Cilag, Lilly, Lundbeck, Medice Arzneimittel Putter, Merz Pharmaceuticals, Novatis Pharma, Pfizer Pharma, Roche, Servier, Shire, some of which are manufacturers of Alzheimer's-disease medication.

\section{References}

Barkley, R.A., 2003. Issues in the diagnosis of attention-deficit/hyperactivity disorder in children. [Review]. Brain \& Development 25, 77-83.

Biederman, J., 1998. Attention-deficit/hyperactivity disorder: a life-span perspective. The Journal of clinical psychiatry 59 (Suppl. 7), 4-16.

Biederman, J., 2005. Attention-deficit/hyperactivity disorder: a selective overview. Biological Psychiatry 57(11), 1215 - 20.

Cao, Q., Zang, Y., Sun, L., Sui, M., Long, X., Zou, Q., Wang, Y., 2006. Abnormal neural activity in children with attention deficit hyperactivity disorder: a resting-state functional magnetic resonance imaging study. Neuroreport 17, 1033-1036. 
Castellanos, F.X., Giedd, J.N., Berquin, P.C., Walter, J.M., Sharp, W., Tran, T., Vaituzis, A.C., Blumenthal, J.D., Nelson, J., Bastain, T.M., Zijdenbos, A., Evans, A.C., Rapoport, J.L, 2001. Quantitative brain magnetic resonance imaging in girls with attention-deficit/hyperactivity disorder. Archives of general psychiatry 58, 289-295.

Castellanos, F.X., Lee, P.P., Sharp, W., Jeffries, N.O., Greenstein, D.K., Clasen, L.S., Blumenthal, J.D., James, R.S., Ebens, C.L., Walter, J.M., Zijdenbos, A., Evans, A.C., Giedd, J.N., Rapoport, J.L, 2002. Developmental trajectories of brain volume abnormalities in children and adolescents with attention-deficit/hyperactivity disorder. The Journal of the American Medical Association 288, 1740-1748.

Castellanos, F.X., Margulies, D.S., Kelly, C., Uddin, L.Q., Ghaffari, M., Kirsch, A., Shaw, D., Shehzad, Z., Di Martino, A., Biswal, B., Sonuga-Barke, E.J., Rotrosen, J., Adler, L.A., Milham, M.P., 2008. Cingulate-precuneus interactions: a new locus of dysfunction in adult attention-deficit/hyperactivity disorder. Biological Psychiatry 63, 332-337.

Catarino, A., Churches, O., Baron-Cohen, S., Andrade, A., Ring, H., 2011. Atypical EEG complexity in autism spectrum conditions: a multiscale entropy analysis. Clinical Neurophysiology 122, 2375-2383.

Conners, C.K., Erhardt, D., Sparrow, E.P., 1999a. Conners’ Adult ADHD Rating Scales (CAARS). North Tonawanda: Multi-Health Systems.

Conners, C.K., Erhardt, D., Epstein, J.N., Parker, J.D.A., Sitarenios, G., Sparrow, E. 1999b. 
Self-ratings of ADHD symptoms in adults I: factor structure and normative data. Journal of Attention Disorders 3,141-51.

Costa, M., Goldberger, A.L., Peng, C.K., 2002. Multiscale entropy analysis of complex physiologic time series. Physical. Review Letters 89(6), 068102.

Erhardt, D., Epstein, J.N., Conners, C.K., Parker, J.D.A., Sitarenios, G., 1999. Self-ratings of ADHD symptoms in adults II: reliability, validity, and diagnostic sensitivity. Journal of Attention Disorders 3, 153-8.

Escudero, J., Abásolo, D., Hornero, R., Espino, P., López, M., 2006. Analysis of electroencephalograms in Alzheimer’s disease patients with multiscale entropy. Physiological Measurement 27, 1091-1106.

Giedd, J.N., Blumenthal, J., Molloy, E., Castellanos, F.X., 2001. Brain imaging of attention deficit/hyperactivity disorder. Annals of the New York Academy of Sciences 931, 3349.

Goldberger, A.L. 1996. Non-linear dynamics for clinicians: Chaos theory, fractals, and complexity at the bedside. The Lancet 347 (9011), 1312-1314.

Goldberger, A.L., 1997. Fractal variability versus pathologic periodicity: Complexity loss and stereotypy in disease. Perspectives in biology and medicine 40 (4), 543-561. 
Goldberger, A.L., Peng, C., Lipsitz, L.A., 2002a. What is physiologic complexity and how does it change with aging and disease? Neurobiology of aging 23(1), 23-26.

Goldberger, A.L., Amaral, L.A.N., Hausdorff, J.M., Ivanov, P.C., Peng, C., Stanley, H.E. 2002b. Fractal dynamics in physiology: Alterations with disease and aging. Proceedings of the National Academy of Sciences of the United States of America 99 (SUPPL. 1), $2466-2472$.

Gomez, C., Poza, J., Garcia, M., Fernandez, A., Hornero, R., 2011. Regularity analysis of spontaneous MEG activity in Attention-Deficit/Hyperactivity Disorder, $33^{\text {rd }}$ Annual International Conference of the IEEE EMBS, August -September, 1765- 1768.

Hill, D.E., Yeo, R.A., Campbell, R.A., Hart, B., Vigil, J., Brooks, W., 2003. Magnetic resonance imaging correlates of attention-deficit/hyperactivity disorder in children. Neuropsychology 17(3), 496-506.

Lake, D.E., Richman, J.S., Pamela Griffin, M., Randall Moorman, J., 2002. Sample entropy analysis of neonatal heart rate variability. American Journal of Physiology - Regulatory Integrative and Comparative Physiology 283 (3), R789-97.

Langleben, D.D., Acton, P.D., Austin, G., Elman, I., Krikorian, G., Monterosso, J.R., Portnoy, O., Ridlehuber, H.W., Strauss, H.W., 2002. Effects of methylphenidate discontinuation on cerebral blood flow in prepubescent boys with attention deficit hyperactivity disorder. Journal of Nuclear Medicine 43, 1624-9. 
Lee, J.S., Kim, B.N., Kang, E., Lee, D.S., Kim, Y.K., Chung, J.K., Lee, M.C., Cho, S.C., 2005. Regional cerebral blood flow in children with attention deficit hyperactivity disorder: comparison before and after methylphenidate treatment. Human Brain Mapping 24, 157-64.

Lipsitz, L.A., 2004. Physiological complexity, aging, and the path to frailty, Science of aging knowledge environment. Science of aging knowledge environment 2004 (16), p. pe 16.

Lou, H.C., Henriksen, L., Bruhn, P., 1990. Focal cerebral dysfunction in developmental learning disabilities. The Lancet 335, 8-11.

Mizuno, T., Takahashi, T., Cho, R.Y., Kikuchi, M., Murata, T., Takahashi, K., Wada, Y., 2010. Assessment of EEG dynamical complexity in Alzheimer's disease using multiscale entropy. Clinical Neurophysiology 121, 1438-1446.

Nichols, T., Hayasaka, S., 2003. Controlling the familywise error rate in functional neuroimaging: A comparative review. Statistical methods in medical research 12(5): 419-446.

Norris, P.R., Canter, J.A., Jenkins, J.M., Moore, J.H., Williams, A.E., Morris, J.A., Jr, 2009. Personalized Medicine: Genetic Variation and Loss of Physiologic Complexity Are Associated With Mortality in 644 Trauma Patients. Annals of surgery 250, 524-530.

Pincus, S.M., 1991. Approximate entropy as a measure of system complexity. Proceedings of the National Academy of Sciences of the United States of America 88 (6), 2297-2301. 
Pincus, S., 1995. Approximate entropy (ApEn) as a complexity measure. Chaos 5(1), 110 117.

Pool, R., 1989. Is it healthy to be chaotic? Science 243 (4891), 604-607.

Protzner, A.B., Valiante, T.A., Kovacevic, N., McCormick, C., McAndrews, M.P., 2010 Hippocampal signal complexity in mesial temporal lobe epilepsy: a noisy brain is a healthy brain. Archive Italiennes de Biologie 148, 289-297.

Raichle, M.E., MacLeod, A.M., Snyder, A.Z., Powers, W.J., Gusnard, D.A., Shulman, G.L., 2001. A default mode of brain function. Proceedings of the National Academy of Sciences of the United States of America 98 (2), 676-82.

Richman, J.S., Moorman, J.R., 2000. Physiological time-series analysis using approximate and sample entropy. American Journal of Physiology - Heart and Circulatory Physiology 278 (6): 47-6.

Rotarska-Jagiela, A., Van de Ven, V., Oertel-Knöchel, V., Uhlhaas, P.J., Vogeley, K., Linden, D.E.J., 2010. Resting-state functional network correlates of psychotic symptoms in schizophrenia. Schizophrenia Research 117, 21-30

Rubia, K., Overmeyer, S., Taylor, E., Brammer, M., Williams, S.C., Simmons, A., Bullmore, E.T., 1999. Hypofrontality in attention deficit hyperactivity disorder during higher-order motor control: a study with functional MRI. The American Journal of Psychiatry 156, 891-896. 
Schulz, K.P., Fan, J., Tang, C.Y., Newcorn, J.H., Buchsbaum, M.S., Cheung, A.M., et al. 2004. Response inhibition in adolescents diagnosed with attention deficit hyperactivity disorder during childhood: an event-related FMRI study. The American Journal of Psychiatry 161, 1650-1657.

Schweitzer, J.B., Lee, D.O., Hanford, R.B., Tagamets, M.A., Hoffman, J.M., Grafton, S.T., Kilts, C.D., 2003. A positron emission tomography study of methylphenidate in adults with ADHD: alterations in resting blood flow and predicting treatment response. Neuropsychopharmacology 28, 967-973.

Seidman, L.J., Biederman, J., Weber, W., Hatch, M., Faraone, S.V., 1998. Neuropsychological function in adults with attention-deficit hyperactivity disorder. Biological Psychiatry 44: 260-8.

Sohn, H., Lee, W., Kim, I., Jeong, J., 2006. Approximate entropy (ApEn) analysis of the EEG in attention-deficit/hyperactivity disorder (AD/HD) during cognitive tasks. Proceedings of the World Congress on Medical Physics and Biomedical Engineering, 1083-1086.

Sokunbi, M.O., Staff, R.T., Waiter, G.D., Ahearn, T.S., Fox, H.C., Deary, I.J., Starr, J.M., Whalley, L.J., Murray, A.D., 2011. Inter-individual Differences in fMRI Entropy Measurements in Old Age. IEEE transactions on bio-medical engineering 58(11), 320614. 
Sun, L., Cao, Q., Long, X., Sui, M., Cao, X., Zhu, C., et al. 2012. Abnormal functional connectivity between the anterior cingulate and the default mode network in drug-naïve boys with attention deficit hyperactivity disorder. Psychiatry Research: Neuroimaging 201, 120-127.

Swanson, J., Castellanos, F.X., Murias, M., LaHoste, G., Kennedy, J., 1998. Cognitive neuroscience of attention deficit hyperactivity disorder and hyperkinetic disorder. Current opinion in neurobiology 8, 263-71.

Takahashi, T., Cho, R.Y., Murata, T., Mizuno, T., Kikuchi, M., Mizukami, K., Kosaka, H., Takahashi, K., Wada, Y., 2009. Age-related variation in EEG complexity to photic stimulation: a multiscale entropy analysis. Clinical Neurophysiology 120, 476-483.

Tamm, L., Menon, V., Ringel, J., Reiss, A.L., 2004. Event-related FMRI evidence of frontotemporal involvement in aberrant response inhibition and task switching in attention deficit/hyperactivity disorder. Journal of the American Academy of Child and Adolescent Psychiatry 43,1430-40.

Tian, L., Jiang, T., Wang, Y., Zang, Y., He, Y., Liang, M., et al. 2006. Altered resting state functional connectivity patterns of anterior cingulated cortex in adolescents with attention deficit hyperactivity disorder. Neuroscience Letters 400, 39-43.

Uddin, L.Q., Kelly, A.M., Biswal, B.B., Margulies, D.S., Shehzad, Z., Shaw, D., Ghaffari, M., Rotrosen, J., Adler, L.A., Castellanos, F.X., Milham, M.P., 2008. Network homogeneity reveals decreased integrity of default-mode network in ADHD. Journal of 
neuroscience methods 169, 249-254.

Wang, L., Zhu, C., He, Y., Zang, Y., Cao, Q., Zhang, H., et al. 2009. Altered small-world brain functional networks in children with attention deficit/hyperactivity disorder. Human Brain Mapping 30(2), 638-49.

Wang, X., Jiao, Y., Lu, Z., 2011. Discriminative Analysis of Resting-state Brain Functional Connectivity patterns of Attention-Deficit Hyperactivity Disorder using Kernel Principal Component Analysis. Eighth IEEE International Conference on Fuzzy Systems and Knowledge Discovery (FSKD) 3, 1938 - 1941.

Wilson, T.W., Franzen, J.D., Heinrichs-Graham, E., White, M.L., Knott, N.L., Wetzel, M.W., 2013. Broadband neurophysiological abnormalities in the medial prefrontal region of the default-mode network in adults with ADHD. Human Brain Mapping 34, 566-574.

Wolf, C., Linden, D.E.J., 2012. Biological pathways to adaptability - interactions between genome, epigenome, nervous system and environment for adaptive behavior. Genes, Brain and Behavior 11, 3-28.

Yang, A.C., Tsai, S.J., Yang, C.H., Kuo, C.H., Chen, T.J., Hong, C.J., 2011. Reduced physiologic complexity is associated with poor sleep in patients with major depression and primary insomnia. Journal of Affective Disorders 131, 179-185. 
Yang, A.C., Huang, C.C., Yeh, H.L., Liu, M.E., Hong, C.J., Tu, P.C., Chen, J.F., Huang, N.E., Peng, C.K., Lin, C.P., Tsai, S.J., 2013 Complexity of spontaneous BOLD activity in default mode network is correlated with cognitive function in normal male elderly: a multiscale entropy analysis. Neurobiology of Aging 34(2), 428-38.

Zang, Y.F., He, Y., Zhu, C.Z., Cao, Q.J., Sui, M.Q., Liang, M., et al., 2007. Altered baseline brain activity in children with ADHD revealed by resting-state functional MRI. Brain \& Development 29, 83-91.

Zweig, M.H., Campbell, G., 1993, Receiver-operating characteristic (ROC) plots: a fundamental evaluation tool in clinical medicine. Clinical Chemistry 39, 561-77. 
Fig. 1: Receiver operating characteristic (ROC) area for discriminating mean whole brain SampEn difference between controls and adult patients with ADHD for $\mathrm{m}=1$ and $\mathrm{m}=2$ and different tolerance values, $\mathrm{r}$.

Fig. 2: 3D whole brain SampEn maps of participants with the same age (21 years). (A) Participant from the ADHD group with mean whole brain SampEn of 1.0481. The arrows point to some areas of low entropy as indicated by the gray scale colour bar $(0$ - 1.4). (B) Participant from the control group with mean whole brain SampEn of 1.2060. Images are displayed in neurological convention.

Fig. 3 (A): depicts the error bars (box plot) of the ADHD and control group. Mean ( $\pm 2 S D)$ SampEn of the ADHD group is $1.04( \pm 0.10)$, while that of the control group is $1.14( \pm 0.07)$, showing a significant difference $(p=0.002)$ in the group mean SampEn of both groups. The ADHD group has lower SampEn when compared to the control group, implying that the control group has more complex fMRI signal. (B) depicts the association between the mean whole brain SampEn and ADHD score of the 30 participants (ADHD and controls). The mean whole brain SampEn of the fMRI signal is negatively correlated with the ADHD scores.

Fig. 4: Surface-rendered images and error bars showing differences between the ADHD patients and control group. Error bar plot depicts the mean SampEn $( \pm 2 S D)$ at the discriminated regions. Rendered images show the difference in SampEn between the ADHD and control group, after correcting for age differences. Regions shown in yellow have lower signal SampEn in the ADHD patients. See Table 2 for a complete list of these regions (threshold $p=0.001, N>250$, corrected cluster $\mathrm{p}<0.05)$. L - Left; R - Right in neurological convention.

Fig. 5: Axial image slices of regions in the brain showing significant negative correlation between the ADHD score and SampEn of both groups. This implies the fMRI signals of the ADHD patients are more predictable (less complex fMRI signal) when compared to the healthy controls. See Table 3 for a list of the peak regions in this image. L - Left; R - Right in neurological convention.

Fig. 6: Shows a significant negative correlation between the ADHD score and SampEn of both groups at; (A) the Middle Occipital Gyrus; (B) the Cuneus; (C) the Middle Temporal Gyrus; (D) the Postcentral Gyrus; (E) the Precuneus; and (F) the Medial Frontal Gyrus. Here the SampEn of the fMRI signal is negatively correlated to the ADHD score. This implies that the higher the ADHD score the lower the SampEn meaning that the ADHD patients have less complex fMRI signal. 
Table 1

Participants characteristics, ADHD score and SampEn

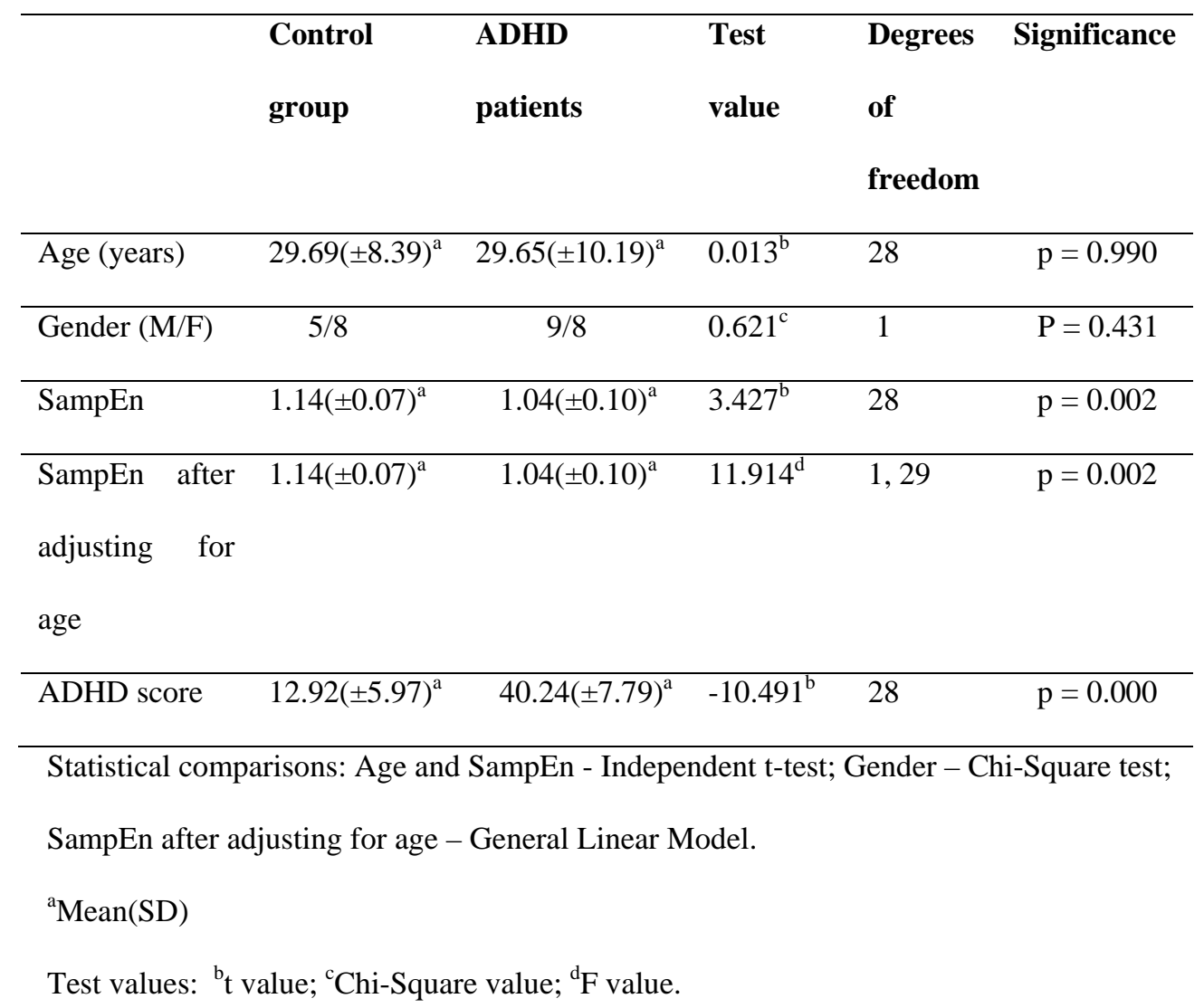


Table 2

Regional differences in signal SampEn between ADHD and Control group (with initial threshold of p=0.001 and family wise error (FWE) corrected cluster level significance of $\mathrm{p}<0.05$ )

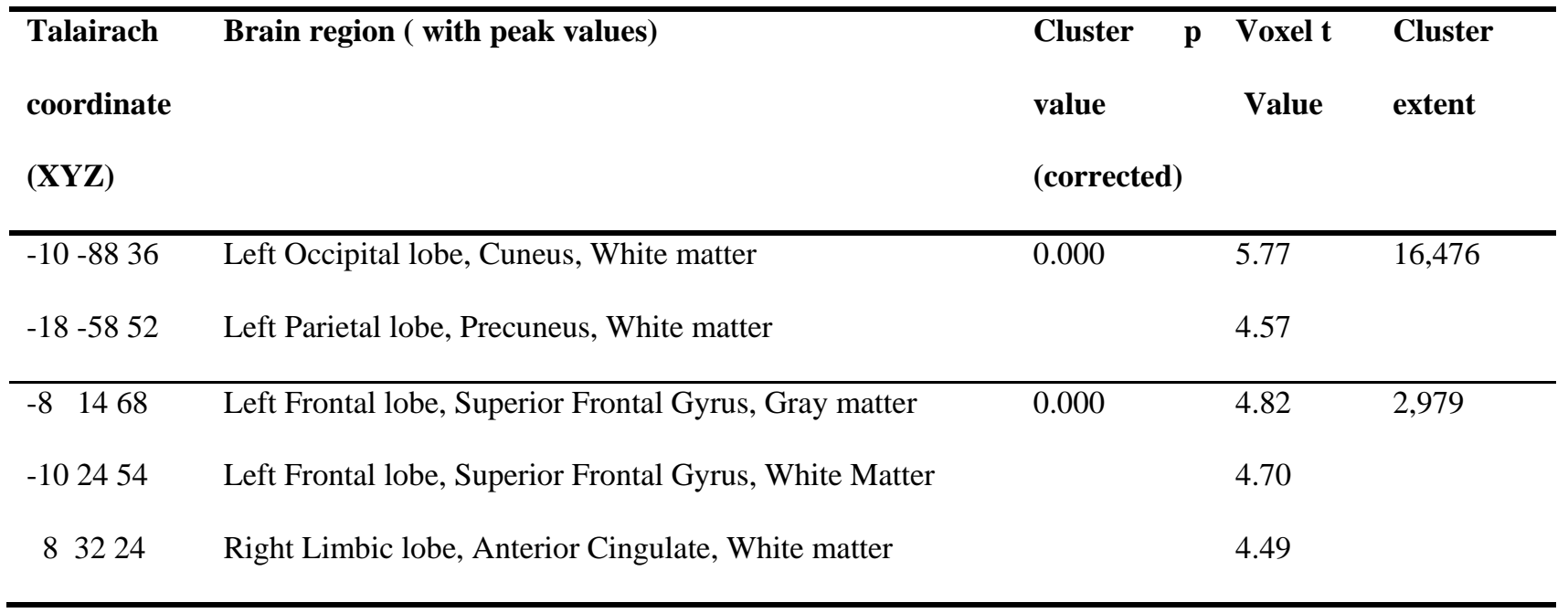


Table 3

Correlation between ADHD score and SampEn (with initial threshold of $\mathrm{p}=0.001$ and family wise error (FWE) corrected cluster level significance of $\mathrm{p}<0.05$ )

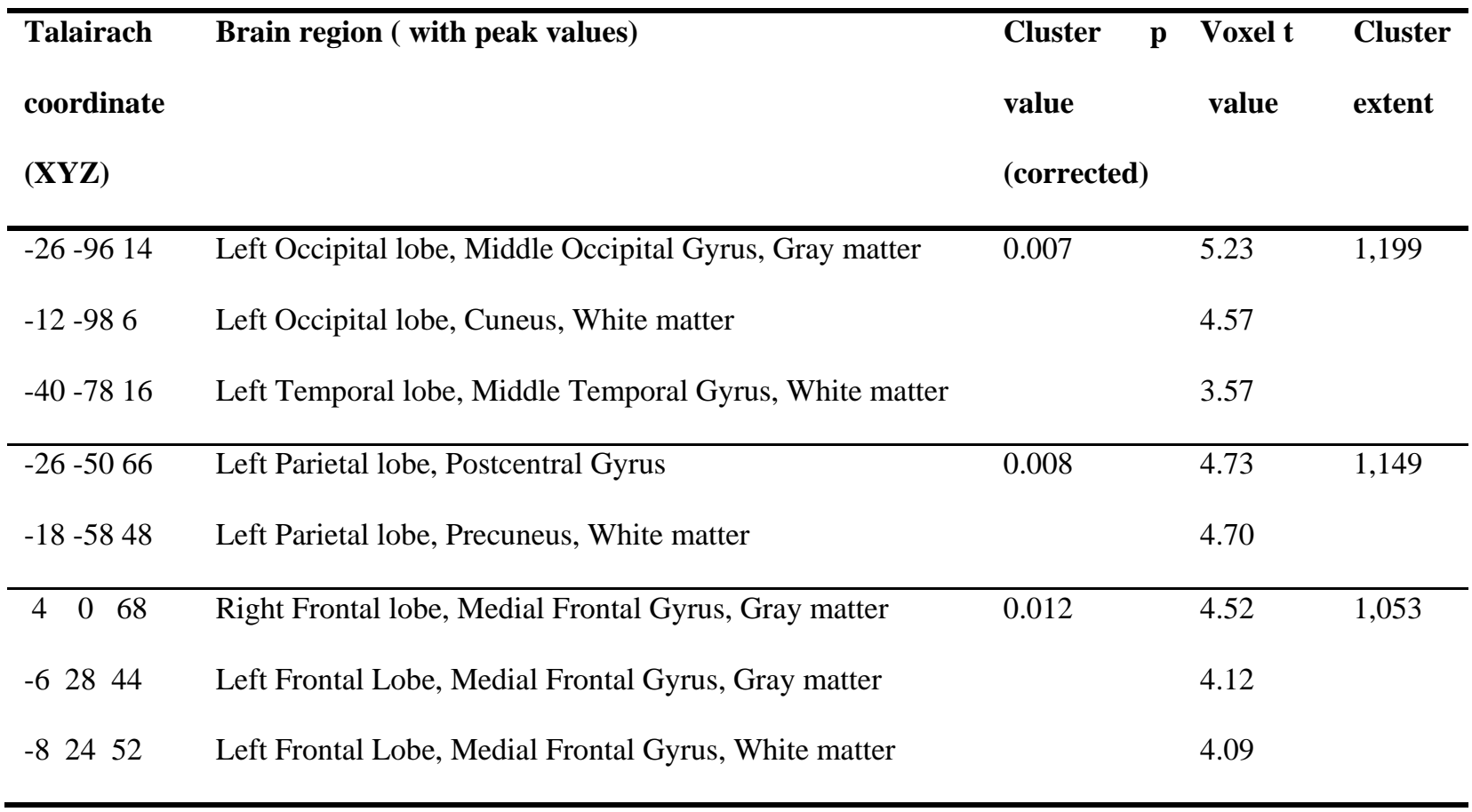




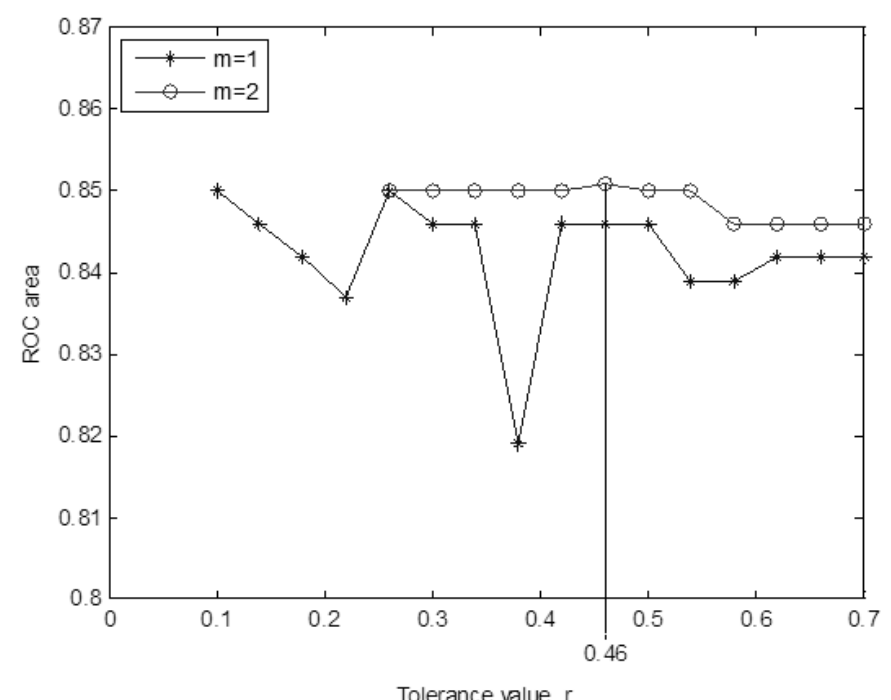




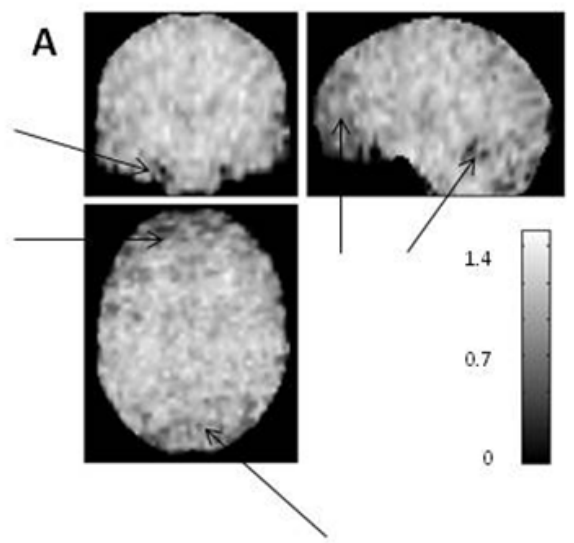

B

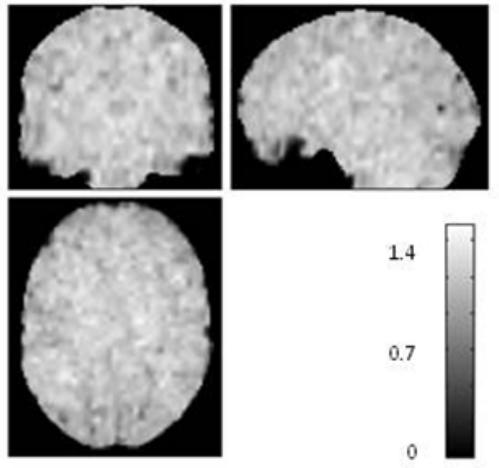



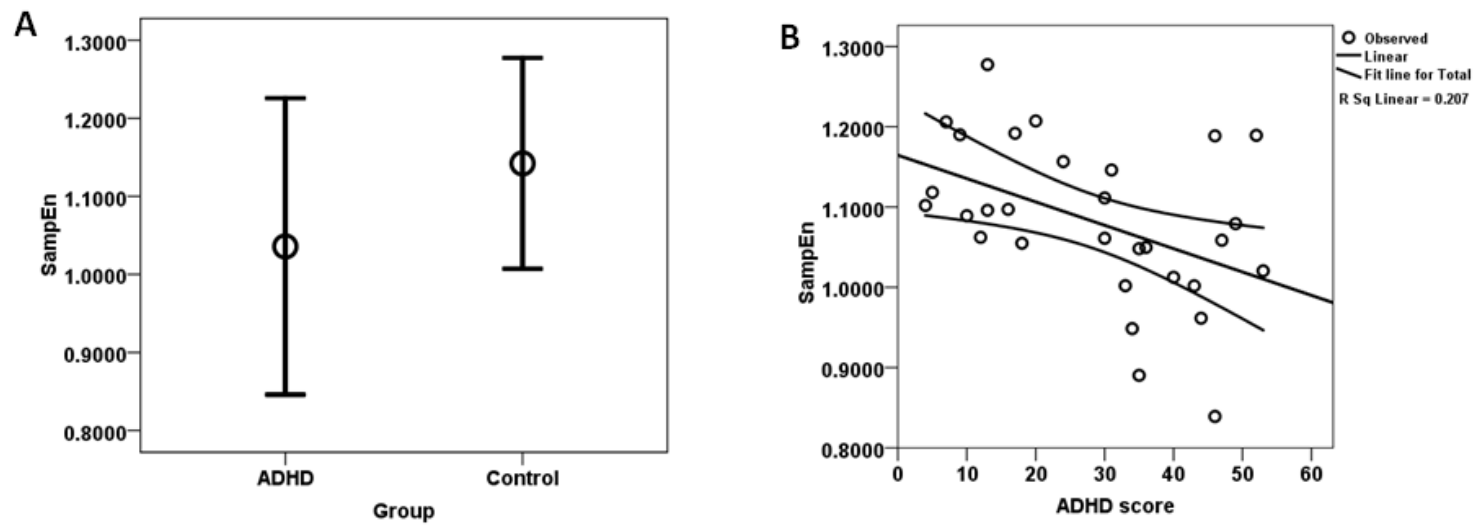


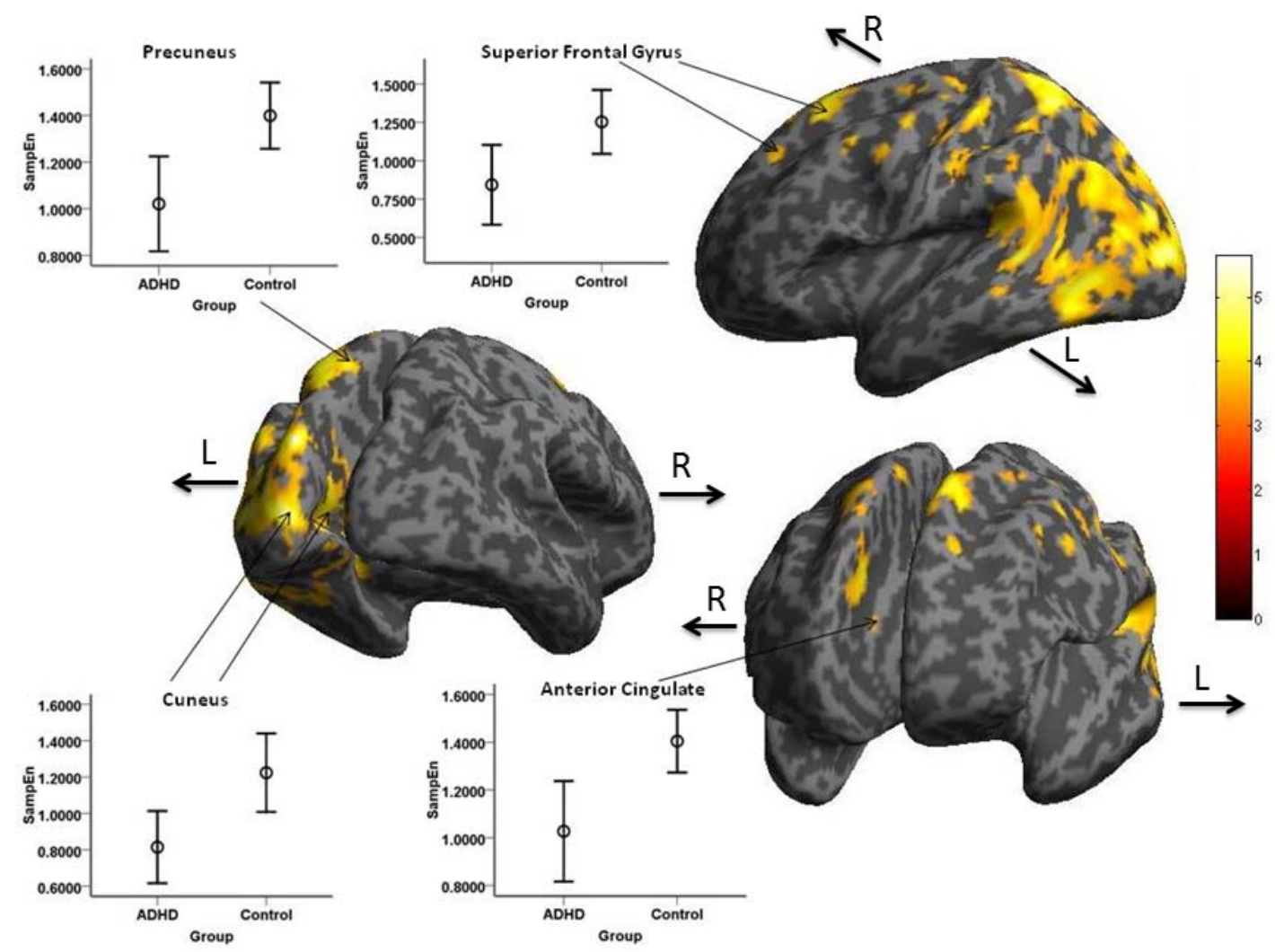




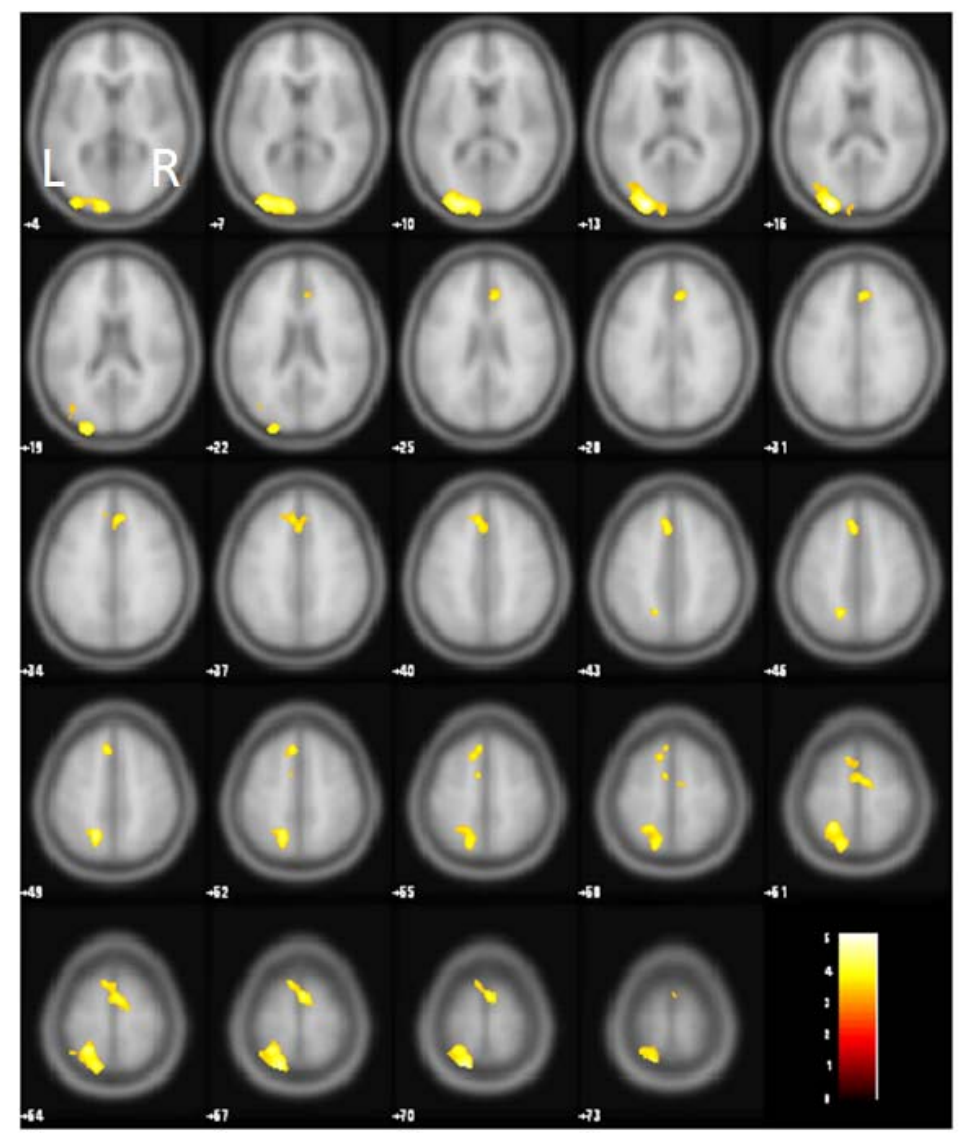




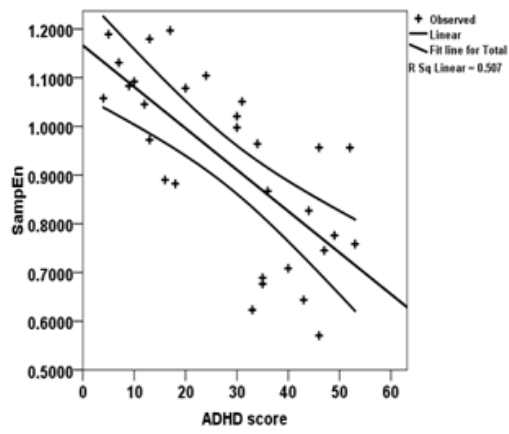

(A) Middle Occipital Gyrus

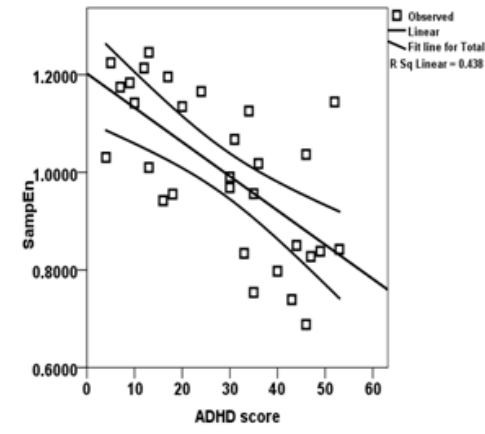

(B) Cuneus

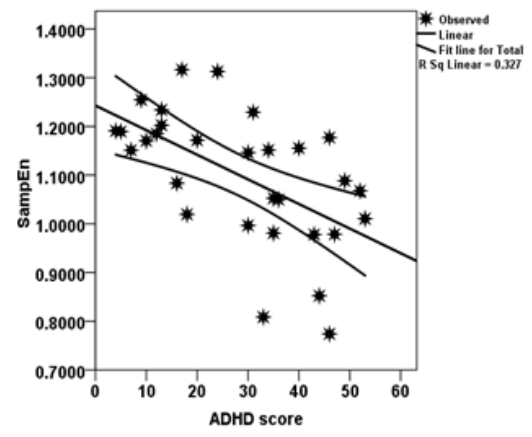

(C) Middle Temporal Gyrus

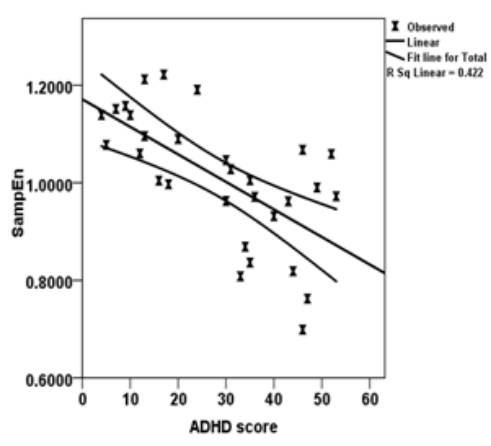

(D) Postcentral Gyrus

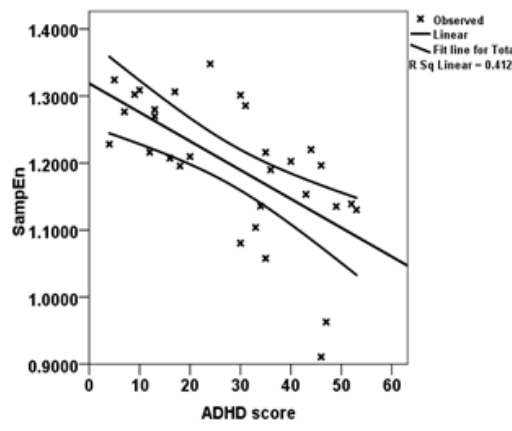

(E) Precuneus

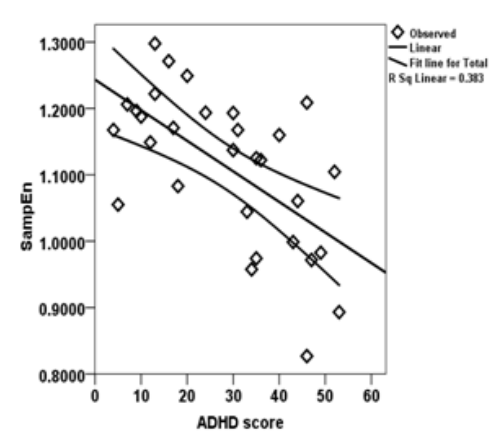

(F) Medial Frontal Gyrus 